\title{
Bid Optimization for Internet Graphical Ad Auction Systems via Special Ordered Sets
}

\author{
Ralphe Wiggins, John A. Tomlin \\ Yahoo!, Inc., 4301 Great America Pkwy, Santa Clara, USA. \\ Email: ralphe@wiggins.name, tomlin@yahoo-inc.com \\ Received November $26^{\text {th }}, 2009$; revised April $3^{\text {rd }}, 2010$; accepted June $10^{\text {th }}, 2010$.
}

\begin{abstract}
This paper describes an optimization model for setting bid levels for certain types of advertisements on web pages. This model is non-convex, but we are able to obtain optimal or near-optimal solutions rapidly using branch and cut opensource software. The financial benefits obtained using the prototype system have been substantial.
\end{abstract}

Keywords: Optimization, Advertising, Electronic Business

\section{Introduction}

Advertising on the World Wide Web is ubiquitous and a big business. Recently a great deal of attention has been paid to "Sponsored Search", where text advertisements with hypertext links are placed next to the search results produced by search engines (see [1]), and these do indeed account for a large fraction of the revenue generated by web advertising. However, a comparable amount of revenue is currently generated by the more traditional graphical advertisements (or more simply, ads) placed on web pages, and that is the type considered in this paper.

We will frequently refer to an Ad Server. This is a machine, or set of machines, which receives HTTP requests for ads when a page is viewed, makes a decision on which ad or ads to display in the ad positions, and returns the ads to the users browser. The algorithms and data which the ad server uses to decide on the ads shown are clearly critical to revenue and profitability.

In the system we are considering (the Yahoo! network), there are two types of ads, or more correctly, ad campaigns. The first of these is known as Class 1 ads, which are sold for a negotiated price to advertisers on a guaranteed basis - that is the ad space (inventory) purchased is guaranteed to be available. The second type is known as Class 2, which is sold by auction, and shown on a "best effort" basis. The Class 2 ads are further divided into House Ads, which Yahoo! purchases for its own use, and the remainder, which are bought by outside advertisers. It is the House ads that will be our major focus here.

One of the problems facing Yahoo!, which acts as publisher, content provider and advertising medium is how to split the Class 2 ad inventory between "house businesses" and paying clients. House businesses (email, personals, etc.) contribute to gross income in 3 ways:

1) Businesses that sell a product or service (i.e., Small Business, Personals) contribute directly to income. The competition with paying clients for inventory resources should be based on net income minus life time revenue reduced by an estimate of the cost of the business.

2) Businesses that enhance traffic by encouraging people to go to other parts of the network. To the extent that the cost of the clicked-on ads is less than the income on the target property, the net income is the difference between these two amounts and such ads can compete in the optimization on that basis. In a dynamic environment, both the cost and income can be tracked to take advantage of current ad effectiveness and user behavior.

3) Finally, there are Yahoo! businesses that enhance income simply by increasing the appeal of visiting the Yahoo! network. While the evaluation of such "appeal" cannot be quantitative, we can use total traffic on each property and a value per user as surrogates.

So long as House businesses can profitably compete for ads under revenue types 1 or 2 , then in principle, and all other things being equal, they should have unlimited budgets since the more they spend, the more Yahoo! makes. However, in reality budgets are not unlimited, and we must accept them as constraints. In addition there are other factors, such as ad fatigue ${ }^{1}$, and the need to deliver the guaranteed Class 1 ads, which limit such 


\section{Class 2 spending.}

We consider setting, or rather re-setting, bids for house Class 2 ads in such a way as to (approximately) maximize expected return for a large group of ads. This involves observing the bid levels of other groups of ads and then re-computing our bids in the relevant auctions in such a way as to maximize expected return for the model horiz- on. This requires choosing among discrete bids, which can be modeled as Special Ordered Sets [2] of type 1. A combination of heuristics applied to the LP solution, and cutting planes, leads to rapid solution of this discrete model.

Use of optimization models for ad campaign planning in the presence of budgets is not completely new, either in the traditional media (see [3]) or in the web context. In the sponsored search setting, Abrams et al. [4] use a linear programming model with column generation to approach the problem. Several papers have attacked the problem of optimizing ad serving in what we have called the Class 1 environment (see [5] and it's references). However, the model discussed here has novel features that require a different approach. The models discussed in [5] and elsewhere assume that the model output can specify the actual serving of specific ads for a page view, that is dictate a serving policy to the ad server. Our only "handle" in the Class 2 context is the bid levels to be set for the auction procedure which then affect the serving policy of the ad server. Clearly this implies some form of discrete model, since a bid either exceeds another bid, or it does not, and the outcome of that will depend on these relative bid levels.

In the remainder of this paper we will outline some of the terminology used with these models, discuss the data available, formulate our model, propose some solution strategies and give computational experience.

\section{Terminology and Data}

Insert order lines (IOs) correspond to the network property/positions where an ad may be placed, i.e., a banner ad across the top, or down the right hand (east) side of a particular page. The number of times this position is available (the number of impressions) is the inventory of this IO we have available for sale.

Business lines in our context are the house businesses seeking to buy some of this inventory. However, Class 2 inventory is sold by auction, not directly, and our only lever to try and increase revenue for house businesses is to set, or reset the bids for the available inventory. Since the house is in competition with outside bidders, we need to take those bids into account.

\footnotetext{
${ }^{1}$ The phenomenon whereby ads become less effective the more they are shown to a user.
}

\section{Ad Server Behavior}

The ad server has quite complicated behavior. For the purposes of this paper it is sufficient to point out that when a request for ads from a page view arrives, a number of factors are considered. Firstly, the candidate ads may be filtered by a user profile requested by the advertiser, which must be compared with the profile of the user (if any). Secondly, Class 1 ads will be shown in preference to Class 2, provided that other factors such as ad fatigue do not interfere. Finally the display of Class 2 ads is determined not only by who "wins" the auction for this property/position, but budget limits and profile matching. Thus the ad corresponding to the highest bidder is not always the one shown. When an ad (and in particular a Class $2 \mathrm{ad}$ ) is shown, the appropriate budget is decremented.

We see from this abbreviated list of characteristics that determining the number of impressions that a Class 2 ad will receive is not a deterministic function of the bids alone. We are therefore reduced to approximating the "value" of an ad with respect to its bid level by using historical data.

\section{Ad Value, Return, and Impressions}

For each Insert Order Line $i$ we define a discrete set of bid levels $b_{i j}$, designed to just exceed the (known) competing bids for the IO. A key concept in our model is the ad value $A_{i j}$ associated with each bid level $j$ for the IO. This is our proxy for the expected amount by which the appropriate budget will be decremented when it gets an impression (i.e., is served up by the ad server). Associated with this ad value is an expected total gross return $L_{i j}$ for making a bid at level $j$ for the IO.

The number of impressions obtained will clearly be influenced by the bid level $j$. Our model requires that we have a relationship between the ad value and return for the IO and the number of impressions expected corresponding to the ad values. An example of such a relationship is shown in Figure 1. The $x$-axis value at the right of each horizontal segment is the expected number of impressions received for the bid level associated with the ad value on the y-axis. These values, denoted $P_{i j}$, along with the $A_{i j}$ and $L_{i j}$ are extrapolated from historical data. Note that the actual bids $b_{i j}$ do not appear themselves in the model below, and we do not discuss the derivation of the ad values, returns, and impression counts further in this paper.

The ad value v. impression graphs are not always as regular as that displayed in Figure 1. More "lopsided" examples are shown in Figures $\mathbf{2}$ and $\mathbf{3}$. 


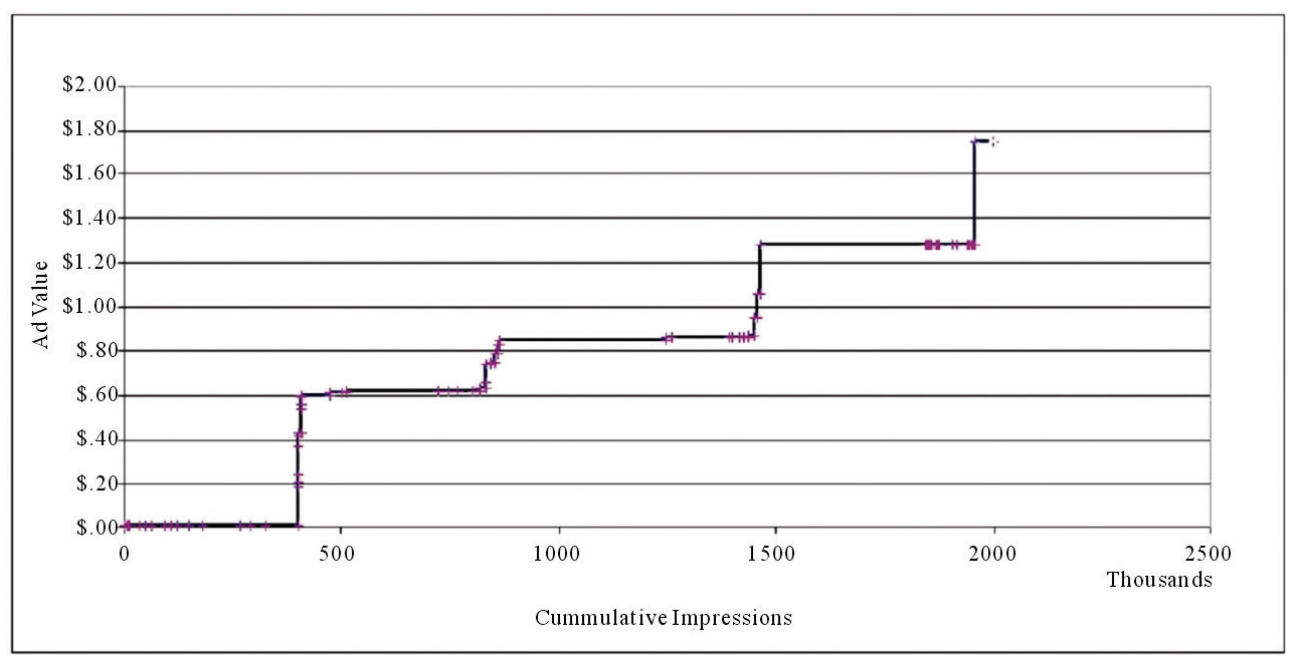

Figure 1. Ad value vs. impressions, Example 1.

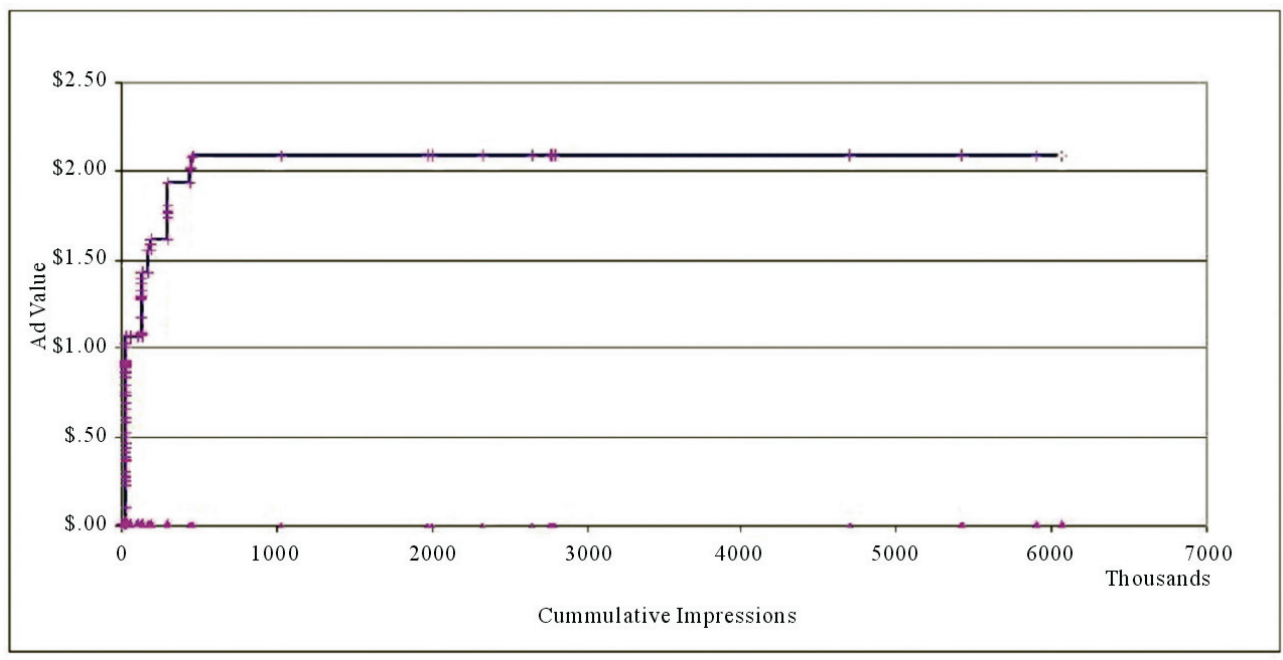

Figure 2. Ad value vs. impressions, Example 2.

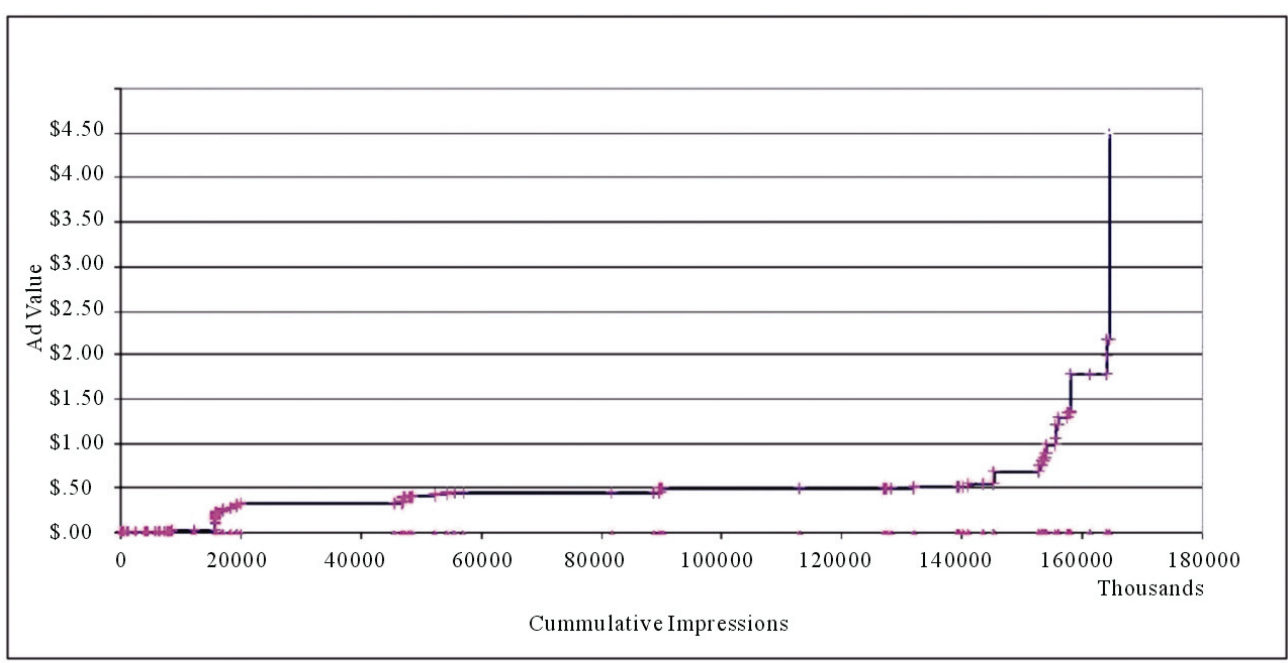

Figure 3. Ad value vs. impressions, Example 3. 


\section{Model Formulation}

We now formally define the optimization model to be solved:

\begin{tabular}{|c|c|}
\hline \multicolumn{2}{|l|}{ Indices } \\
\hline$i=1, \ldots, I$ & The insert order lines \\
\hline$j=1, \ldots, J_{i}$ & The bid estimate levels for line $i$ \\
\hline$k=1, \ldots, B L$ & The business lines \\
\hline \multicolumn{2}{|l|}{ Data } \\
\hline$I_{k}$ & The subset of order lines for business line $k$ \\
\hline$B_{k}$ & Budget for business line $k$ \\
\hline$L_{i j}$ & Return for line $i$, estimate $j$ \\
\hline$A V_{i j}$ & Ad value associated with pair $(i, j)$ \\
\hline$P_{i j}$ & Number of impressions for combination $(i, j)$ \\
\hline$C T R_{i k}$ & $\begin{array}{l}\text { Expected click-through rate for business line } k \text { on } \\
\text { insert line } i\end{array}$ \\
\hline$C P C_{k}$ & (Given) cost per click for business line $k$ \\
\hline$V$ & Overall impression budget for House Class 2 \\
\hline \multicolumn{2}{|l|}{ Variables } \\
\hline$\delta_{i j}$ & $\begin{array}{l}\text { has value } 1 \text { if level } j \text { chosen for line } i, 0 \text { other- } \\
\text { wise }\end{array}$ \\
\hline
\end{tabular}

Constraints SOS1 (multiple choice)

$$
\sum_{j} \delta_{i j}=1 \quad \forall i
$$

Budgets

$$
\sum_{i \in I_{k}} \sum_{j} P_{i j} \cdot A V_{i j} \delta_{i j} \leq B_{k} \quad \forall k
$$

Click Values

$$
\sum_{i \in I_{k}} \sum_{j} P_{i j} \cdot A V_{i j} \delta_{i j} \leq C P C_{k} \cdot \sum_{i \in I_{k}} \sum_{j} C T R_{i k} \cdot P_{i j} \delta_{i j} \quad \forall k
$$

Impression Budget

$$
\sum_{k} \sum_{i \in I_{k}} \sum_{j} P_{i j} \delta_{i j} \leq V
$$

Objective

Maximize $\quad \sum_{k} \sum_{i \in I_{k}} \sum_{j} L_{i j} \delta_{i j}$

The purpose of the $\delta_{i j}$ variables is to choose a bid level from the finite set of possibilities presented for IO line $i$. Following the description in Section 4, we see that the ad value for an insert line $i$ is therefore $\sum_{j} A V_{i j} \delta_{i j}$, the return is $\sum_{j} L_{i j} \delta_{i j}$ and the expected number of impressions is $\sum_{j} P_{i j} \delta_{i j}$. We also insert a "do nothing" variable $\delta_{i 0}$, or explicit slack, at the beginning of each set. Note that by definition at most one of variables which make up a SOS1 (special ordered set [2] of type 1) may be nonzero, and in this case the constraint (1) implies that the SOS1 variables must be zero or one in a valid solution, and therefore integer.

Note that except for the overall impression constraint
(4), the model falls into disjoint sub models, one for each Business line $k$. This loose connection makes the model somewhat easier to solve than we might expect for a non-convex model, especially since it may often be nonbinding. This would allow solution of a sequence of independent models. However, the later case is hard to predict a priori and in any case the size of the overall model has so far proved easily manageable.

\section{Implementation and Solution Strategy}

The model is generated and solved using a suite of programs. The data on the advertising campaigns and budgets are retrieved from an Oracle data base via an SQL program, which feeds them to a $\mathrm{C}$ program that generates a standard MPS data file. This is read by the solver, which is built on the COIN-OR open-source $\mathrm{C}++$ library [6]. In particular we use the Special Ordered Set capabilities of the Coin Branch-and-Cut (CBC) library[7], using a strategy to be discussed below. When a satisfactory solution is obtained it is written to file in pseudoMPS output format, for use by another $\mathrm{C}$ program which interprets the solution for the bidding software.

One advantage of using this implementation strategy is that it is very easy to design solution strategies which limit the branch and cut search. Since we have introduced several layers of approximation in the formulation of the model, and the derivation of its data, it would be foolish to insist on achieving an exact optimum. Thus a first feasible integer solution is perfectly adequate, provided the integer "gap" is small enough, and we may hope to use simple heuristics to give the search a hot start.

Some familiarity with branch and bound, branch and cut and special ordered sets will be assumed in the remainder of this section, but the reader who is only interested in the results can skip to Section 8.

We experimented with 2 hot start strategies:

1) When an SOS is exactly, or almost, satisfied in the LP solution, i.e., one member of the set is close to 1 , which is most of the time, that member is fixed to 1 , and the other members fixed to zero, provided either that (a) this member is the first member of the set, or (b) the reduced costs of the other members are greater than some tolerance.

2) If exactly one member of a set is nonzero, it is fixed to 1 and all other members to zero. Otherwise all members up to the first nonzero, and after the last nonzero are fixed to zero.

There is a slight possibility that these variable fixing strategies will make the problem integer infeasible, in which case we would have to relax them again. We return to this point later on.

In addition to this fixing of variables we apply 3 of the types of "cuts" available in the CBC library — known as 
"Probing", "Gomory" and "Knapsack" cuts. If Strategy 2 is used we also add "Redsplit" and "Clique" cuts.

Tables 1 and 2 give the results of running some representative problems with the two strategies. The results are given in terms of percentage degradation of the first integer solution found from the continuous LP solution, and the times taken on an Intel Linux box (with Xeon 2.8 $\mathrm{GHz}$ processor and $2 \mathrm{~GB}$ of RAM). The "best" known solution is that found within 1200 seconds. In most cases, we were able to prove optimality of the first solution, subject to the variable fixing that had been carried out (hence the slight differences in the best known solutions for the 2 strategies). However, Strategy 1 was clearly not satisfactory for problem 6 , though it solved easily with Strategy 2.

In general we conclude that both strategies may become too aggressive as models become larger and more complex. Even if the times are acceptable (we expect to solve this daily, or at most hourly), the degradations can become poor.

\section{Relaxation to SOS2}

One approach to the problems seen above is to relax the model. If we consider the relationships expressed in Figures 1-3 we see that there is no advantage to having an ad value on the vertical segments of the graphs, unless this allows us to maintain budget feasibility. Maintaining this feasibility is the biggest cause of degradation from the LP solution, furthermore experience shows that this is an issue in only a tiny fraction of the lines in the model. We therefore cavalierly dispense with the SOS1 re-

Table 1. Degradations of first integer solutions, Strategy 1.

\begin{tabular}{ccccc}
\hline & Number & Strategy 1 & Time & Best known \\
\hline Model & of SOS & \% degradation & (seconds) & \% degradation \\
\hline 1 & 2704 & 2.04 & 0.239 & 2.04 \\
2 & 5508 & 2.94 & 10.33 & 2.87 \\
3 & 6589 & 6.91 & 0.572 & 6.91 \\
4 & 8410 & 18.94 & 0.721 & 18.94 \\
5 & 11504 & 6.99 & 44.99 & 6.99 \\
6 & 16259 & $? ? ? ?$ & $>1200$ & $? ? ? ?$ \\
\hline
\end{tabular}

Table 2. Degradations of first integer solutions, Strategy 2.

\begin{tabular}{ccccc}
\hline & Number & Strategy 2 & Time & Best known \\
\hline Model & of SOS & \% degradation & (seconds) & \% degradation \\
1 & 2704 & 2.167 & 0.107 & 2.167 \\
2 & 5508 & 3.035 & 0.215 & 3.035 \\
3 & 6589 & 6.929 & 0.268 & 6.929 \\
4 & 8410 & 19.345 & 0.356 & 19.345 \\
5 & 11504 & 9.062 & 0.455 & 9.062 \\
6 & 16259 & 8.346 & 8.214 & 8.364 \\
\hline
\end{tabular}

quirement that only one member of a set be non-zero, but allow at most two members of the set to be nonzero, and then only if they are adjacent-in other words relax the SOS1 set to a SOS2 set (see [8]). This will have no effect for most of the sets, but allow us to "fudge" borderline cases.

Following this relaxation, we adopt a simpler hot start procedure for the now non-convex (not integer) tree search. Firstly, the integer cuts must be dispensed with. Secondly, our variable fixing procedure simply looks at the LP solution, and for each set, flags to zero those variables before the first non-zero member, and those after the last non-zero member. If a set is not satisfied we attempt a temporary fixing of all the variables not so far fixed, except the two which define the "current interval" as defined in $[2,8]$. The LP is then resolved. If it is feasible this process will have led to a valid - one hopes, good-solution, which may be used to put a bound on the valid solutions. If not, we obtain no such bound. The variables which were temporarily fixed are now unfixed, and we proceed to the branch and bound algorithm. This strategy, which we call Strategy 3, has been more consistent than use of SOS1, and the one we use in practice. Results for the same set of problems as in Table $\mathbf{1}$ are shown in Table 3. In the rare cases when an SOS2 set is satisfied with 2 non-zero members we simply use the interpolated bid.

Because of the relaxation, the degradations are significantly smaller than with SOS1, as expected, but this should not be considered very significant.

\section{Practical Results}

Participation in this program, as opposed to continuing with the traditional manual process, is on an opt-in basis for each House business. It is gratifying that more and more of these businesses have opted in, but perhaps not surprising, since the model attempts to optimize the portfolio of IOs for each business, rather than treating them independently and greedily. Without giving company confidential dollar figures, we can indicate the growing practical success of our model by comparing the Return on Investment (ROI) achieved by the businesses which have opted in compared with those that have not.

Table 3. Degradations of first SOS2 solutions, Strategy 3.

\begin{tabular}{ccccc}
\hline & Number & Strategy 3 & Time & Best known \\
\hline Model & of SOS & \% degradation & (seconds) & \% degradation \\
1 & 2704 & 0.270 & 0.809 & 0.270 \\
2 & 5508 & 0.561 & 1.203 & 0.561 \\
3 & 6589 & 0.126 & 1.834 & 0.126 \\
4 & 8410 & 0.038 & 3.218 & 0.038 \\
5 & 11504 & 0 & 3.958 & 0 \\
6 & 16259 & 0.500 & 113.2 & 0.498 \\
\hline
\end{tabular}


Table 4. ROI for model vs. manual.

\begin{tabular}{ccc}
\hline & Model & Manual \\
\hline Quarter & ROI & ROI \\
Q4 2005 & 0.90 & 0.59 \\
Q1 2006 & 1.36 & 0.89 \\
Q2 2006 & 1.98 & 0.37 \\
Q3 2006 & 0.59 & 0.34 \\
Q4 2006 & 1.72 & -0.28 \\
Q1 2007 & 1.39 & -0.39 \\
Average & 1.32 & 0.25 \\
\hline
\end{tabular}

ROI is computed as the ratio of the imputed income to the delivery cost, minus 1 . The RIOS achieved by the model and the manual process are shown in Table 4 for the period Q4 2005 through Q1 2007.

In general we conclude that both strategies may become too aggressive as models become larger and more complex. Even if the times are acceptable (we expect to solve this daily, or at most hourly), the degradations can become poor.

\section{Conclusions}

The problem of setting bid levels for impressions of Class 2 ads can not only be expressed in terms of a non-convex optimization problem, but efficiently solved to satisfactory accuracy, and the solutions implemented by an ad server which accepts such bids as a basis for its serving decisions. This enables us to increase both efficiency of the process and profitability of the outcome. Due to the success of the pilot model we have described, which has been in operation for some time, it has now been transformed into an official company "product" to handle Class 2 ads.

\section{Acknowledgements}

We are indebted to our colleagues Ryan Christensen,
Andrea Ford, and Madhu Vudali for their encouragement and cooperation in the course of this work.

\section{REFERENCES}

[1] B. Edelman, M. Ostrovsky and M. Schwarz, "Internet Advertising and the Generalizaed Second Price Auction: Selling Billions of Dollars Worth of Keywords," 2nd Workshop on Sponsored Search Auctions, Ann Arbor, June 2006.

[2] E. M. L. Beale and J. A. Tomlin, "Special Facilities in a General Mathematical Programming System for NonConvex Problems Using Ordered Sets of Variables," In: J. Lawrence, Ed., Proceedings 5th IFORS Conference, Tavistock, London \& Wiley, New York, 1970, pp. 447-454.

[3] S. Bollapragada, H. Chen, M. Phillips and M. Garbiras, M. Scholes, T. Gibbs and M. Humphreville, " $N B C$ 's Optimization Systems Increase Revenues and Productivity," Interfaces, Vol. 32, No. 1, 2002, pp. 47-60.

[4] Z. Abrams, O. Mendelevitch and J. A. Tomlin, "Optimal Delivery of Sponsored Search Advertisements Subject to Budget Constraints," Proceedings of ACM EC'07, San Diego, June 2007.

[5] N. Abe, "Improvements to the Linear Programming Based Scheduling of Web Advertisements," Journal of Electronic Commerce Research, Vol. 5, No. 1, 2005, pp. 75-98.

[6] R. Lougee-Heimer, F. Barahona, B. L. Dietrich, J. P. Fasano, J. J. Forrest, R. Harder, L. Ladanyi, T. Pfender, T. Ralphs, M. Saltzman and K. Scheinberg, "The COIN$O R$ Initiative: Accelerating Operations Research Progress through Open-Source Software," ORMS Today, Vol. 28, No. 5, 2001, pp. 48-49.

[7] COIN-OR Foundation. http://www.coin.or.org

[8] J. Forrest and J. Tomlin, "Branch and Bound, Integer, and Non-Integer Programming," Annals of Operations Research, Vol. 149, No. 1, 2007, pp. 81-87. 